EPJ manuscript No.

(will be inserted by the editor)

\title{
Conductivity of disordered graphene at half filling
}

\author{
P. M. Ostrovsky ${ }^{1, a}$, I. V. Gornyi ${ }^{1, b}$, and A. D. Mirlin ${ }^{1,2, c}$ \\ 1 Institut für Nanotechnologie, Forschungszentrum Karlsruhe, 76021 Karlsruhe, Germany \\ ${ }^{2}$ Institut für Theorie der kondensierten Materie, Universität Karlsruhe, 76128 Karlsruhe, Germany
}

\begin{abstract}
We study electron transport properties of a monoatomic graphite layer (graphene) with different types of disorder at half filling. We show that the transport properties of the system depend strongly on the symmetry of disorder. We find that the localization is ineffective if the randomness preserves one of the chiral symmetries of the clean Hamiltonian or does not mix valleys. We obtain the exact value of minimal conductivity $4 e^{2} / \pi h$ in the case of chiral disorder. For long-range disorder (decoupled valleys), we derive the effective field theory. In the case of smooth random potential, it is a symplectic-class sigma model including a topological term with $\theta=\pi$. As a consequence, the system is at a quantum critical point with a universal value of the conductivity of the order of $e^{2} / h$. When the effective time reversal symmetry is broken, the symmetry class becomes unitary, and the conductivity acquires the value characteristic for the quantum Hall transition.
\end{abstract}

\section{Introduction}

Almost 50 years ago, in a pathbreaking paper "Absence of diffusion in certain random lattices" [1] Anderson demonstrated that a quantum particle may become localized by a random potential. In particular, in non-interacting systems of two-dimensional (2D) geometry even weak disorder localizes all electronic states [2], thus leading to the exactly zero conductivity, $\sigma=0$.

Recent breakthrough in graphene fabrication [4] and subsequent transport experiments [567/89 revealed remarkable electronic properties of this material [10]11. One of the most striking experimental observation is the minimal conductivity $\sigma$ of order $e^{2} / h$ observed in undoped samples and staying almost constant in a wide range of temperatures $T$ from $300 \mathrm{~K}$ down to $\sim 1 \mathrm{~K}$. This behavior should be contrasted to well-established results on the conductivity of $2 \mathrm{D}$ systems where Anderson localization drives the system into insulating state at low $T$ 2]312]13/14. An apparently $T$-independent value of $\sigma \sim e^{2} / h$ suggests that the system is close to a quantum critical point and calls for a theoretical explanation. The aim of this paper is to analyze what one should expect for conductivity from the theoretical point of view. We will see that, in view of the unconventional character of the graphene spectrum, the nature of disorder is crucially important.

In this paper we consider the conductivity of a single-layer graphene at half filling, $\varepsilon=0$. The Drude conductivity [15]16] has the value $\sigma=4 e^{2} / \pi h$ at this point. Since this value is of the order of conductance quantum, the localization effects should, in principle, become strong at half filling. At lowest temperatures $T$, the system with generic disorder preserving time-reversal symmetry falls into the orthogonal symmetry class [12 13/14 implying strong localization regime [13. In this situation, the conductivity at half filling should have a pronounced temperature

${ }^{a}$ Also at L. D. Landau Institute for Theoretical Physics RAS, 119334 Moscow, Russia.

b Also at A.F. Ioffe Physico-Technical Institute, 194021 St. Petersburg, Russia.

c Also at Petersburg Nuclear Physics Institute, 188300 St. Petersburg, Russia. 
dependence and get strongly suppressed with lowering $T$. This conclusion remains valid for generic disorder also if the time-reversal symmetry is broken (e.g. by magnetic impurities), so that the symmetry is unitary [14. Remarkably, this is not what is observed in the experiment 56. According to what was said above, this might only happen, if at all, for a particular type of disorder.

We identify two broad classes of randomness in graphene - chiral disorder (preserving chiral symmetry of clean graphene) and long-range disorder (not mixing the two valleys) — leading to the lack of localization and emergence of quantum criticality and associated universal minimal conductivity [16 17].

\section{The Model}

The tight-binding Hamiltonian of clean graphene is a $4 \times 4$ matrix operating in the AB space of two sublattices (Pauli matrices $\sigma_{i}$ ) and in the $K-K^{\prime}$ space of the valleys (Pauli matrices $\tau_{i}$ ). Therefore we introduce the four-component wave function

$$
\Psi=\left\{\phi_{A K}, \phi_{B K}, \phi_{B K^{\prime}}, \phi_{A K^{\prime}}\right\}^{T}
$$

In this representation the Hamiltonian has the form

$$
H=v_{0} \tau_{3} \boldsymbol{\sigma} \mathbf{k} .
$$

Here $\tau_{3}$ is the third Pauli matrix in the $K-K^{\prime}$ space and $\boldsymbol{\sigma}=\left\{\sigma_{1}, \sigma_{2}\right\}$ is the two-dimensional vector of Pauli matrices in the $\mathrm{AB}$ space. The Fermi velocity in graphene is $v_{0} \simeq 10^{8} \mathrm{~cm} / \mathrm{s}$. In fact, the form of the Hamiltonian (2) is universal and does not rely on the tight-binding approximation. The degeneracy of the spectrum in $K$ and $K^{\prime}$ points is provided by the twodimensional representation of the honeycomb lattice symmetry group, while the expression (2) is the first-order $k$-expansion near these points.

Let us analyze the symmetries of the clean graphene Hamiltonian (2). First, the system is obviously uniform and isotropic. Any disorder considered in this paper preserves these symmetries on average, so we do not pay much attention to them here. Second, due to the two valley structure of the electron spectrum the whole $\mathrm{SU}(2)$ symmetry group exists in an isospin space of the valleys. The generators of this group are [12]

$$
\Lambda_{x}=\sigma_{3} \tau_{1}, \quad \Lambda_{y}=\sigma_{3} \tau_{2}, \quad \Lambda_{z}=\sigma_{0} \tau_{3}
$$

These three operators commute with the Hamiltonian and anticommute with each other. Third, there are two more relevant symmetries of the clean Hamiltonian, namely, time inversion operation (we denote it $T_{0}$ ) and chiral symmetry $\left(C_{0}\right)$. Using various combinations of $T_{0}, C_{0}$, and rotations of the isospin $\left(\Lambda_{0, x, y, z}\right)$ one can easily construct a whole bunch of twelve symmetry operations

$$
\begin{array}{lll}
T_{0}: A \mapsto \sigma_{1} \tau_{1} A^{T} \sigma_{1} \tau_{1}, & C_{0}: A \mapsto-\sigma_{3} \tau_{0} A \sigma_{3} \tau_{0}, & C T_{0}: A \mapsto-\sigma_{2} \tau_{1} A^{T} \sigma_{2} \tau_{1}, \\
T_{x}: A \mapsto \sigma_{2} \tau_{0} A^{T} \sigma_{2} \tau_{0}, & C_{x}: A \mapsto-\sigma_{0} \tau_{1} A \sigma_{0} \tau_{1}, & C T_{x}: A \mapsto-\sigma_{1} \tau_{0} A^{T} \sigma_{1} \tau_{0}, \\
T_{y}: A \mapsto \sigma_{2} \tau_{3} A^{T} \sigma_{2} \tau_{3}, & C_{y}: A \mapsto-\sigma_{0} \tau_{2} A \sigma_{0} \tau_{2}, & C T_{y}: A \mapsto-\sigma_{1} \tau_{3} A^{T} \sigma_{1} \tau_{3}, \\
T_{z}: A \mapsto \sigma_{1} \tau_{2} A^{T} \sigma_{1} \tau_{2}, & C_{z}: A \mapsto-\sigma_{3} \tau_{3} A \sigma_{3} \tau_{3}, & C T_{z}: A \mapsto-\sigma_{2} \tau_{2} A^{T} \sigma_{2} \tau_{2} .
\end{array}
$$

Note that the transposition changes sign of momentum operators.

Now we incorporate a disorder of the form $V_{i j} \sigma_{i} \tau_{j}$ in the model, breaking some of the above symmetries. The average isotropy of the disordered graphene implies that $\Lambda_{x}$ and $\Lambda_{y}$ symmetries of the Hamiltonian are present or absent simultaneously. Below we combine them into a single notation $\Lambda_{\perp}$, and proceed in the same way with $T_{\perp}$ and $C_{\perp}$. In Table 1 we list all possible matrix structures of disorder along with their symmetries.

Generally, the chiral symmetry implies that the Hamiltonian takes block-off-diagonal form under a proper unitary transformation. For instance, a generic disorder preserving $C_{z}$ symmetry 
Table 1. Symmetries of various disorders in graphene. The first five rows of the table contain disorders preserving physical time inversion symmetry $T_{0}$. The next four present structures violating $T_{0}$. Note that each row contains "+" either in the $\Lambda_{z}$ column or in one of the $C$ columns. This means that each disorder structure from the first column - when taken alone — produces a finite conductivity at the Dirac point.

\begin{tabular}{cccccccccccc}
\hline \hline Disorder structure & $\Lambda_{\perp}$ & $\Lambda_{z}$ & $T_{0}$ & $T_{\perp}$ & $T_{z}$ & $C_{0}$ & $C_{\perp}$ & $C_{z}$ & & $C T_{0}$ & $C T_{\perp} C T_{z}$ \\
\hline$\sigma_{0} \tau_{0}$ & + & + & + & + & + & - & - & - & - & - & - \\
$\sigma_{\{1,2\}} \tau_{\{1,2\}}$ & - & - & + & - & - & + & - & - & + & - & - \\
$\sigma_{1,2} \tau_{0}$ & - & + & + & - & + & + & - & + & + & - & + \\
$\sigma_{0} \tau_{1,2}$ & - & - & + & - & - & - & - & + & - & - & + \\
$\sigma_{3} \tau_{3}$ & - & + & + & - & + & - & + & - & - & + & - \\
\hline$\sigma_{3} \tau_{1,2}$ & - & - & - & - & + & - & - & + & + & - & - \\
$\sigma_{0} \tau_{3}$ & - & + & - & + & - & - & + & - & + & - & + \\
$\sigma_{1,2} \tau_{3}$ & + & + & - & - & - & + & + & + & - & - & - \\
$\sigma_{3} \tau_{0}$ & + & + & - & - & - & - & - & - & + & + & + \\
\hline \hline
\end{tabular}

Table 2. Possible types of disorder in graphene leading to a finite minimal conductivity. Each row of the table corresponds to a certain universality class of the problem. In the first column we give an example of the disorder that belongs to this class (RMF is an external random magnetic field). The relevant symmetries are placed in the second column. The symmetry class itself is indicated in the third column according to the classification of Altland and Zirnbauer [19|20|21. The fourth column displays the dominant non-trivial term in the corresponding sigma model. This term is responsible for the absence of localization. There are three possibilities 22]: (i) Gade term 24 23, (ii) Wess-Zumino-Witten term 25121, or (iii) Pruisken $\theta$-term [26] with $\theta=\pi$. The value of the minimal conductivity is given in the last column. The first five rows contain chiral-symmetric types of disorder [ripples $\left(\sigma_{1,2} \tau_{0}\right)$ can be added to any of them]. They all yield the minimal conductivity $4 e^{2} / \pi h$ (up to power-law corrections in a weak disorder strength for $C_{z}$ chirality, hence " $\approx$ "), see Sec. 3 The last four rows correspond to the case of decoupled valleys (long-range disorder), see Sec. 4 from top to bottom: random Dirac vector potential, scalar potential, mass, and any of their combinations. The value of the minimal conductivity depends on a particular symmetry of the long-range disorder.

\begin{tabular}{ccccc}
\hline \hline Disorder & Symmetries & Class & Sigma model & Conductivity \\
\hline Vacancies, strong potential impurities & $C_{z}, T_{0}$ & BDI & Gade & $\approx 4 e^{2} / \pi h$ \\
Vacancies + RMF & $C_{z}$ & AIII & Gade & $\approx 4 e^{2} / \pi h$ \\
$\sigma_{3} \tau_{1,2}$ disorder & $C_{z}, T_{z}$ & CII & Gade & $\approx 4 e^{2} / \pi h$ \\
Dislocations & $C_{0}, T_{0}$ & CI & WZW & $4 e^{2} / \pi h$ \\
Dislocations + RMF & $C_{0}$ & AIII & WZW & $4 e^{2} / \pi h$ \\
\hline Ripples, RMF & $\Lambda_{z}, C_{0}$ & $2 \times$ AIII & WZW & $4 e^{2} / \pi h$ \\
\hline Charged impurities & $\Lambda_{z}, T_{\perp}$ & $2 \times \mathrm{AII}$ & $\theta=\pi$ & $4 \sigma_{S p}^{* *}$ \\
random Dirac mass: $\sigma_{3} \tau_{0}, \sigma_{3} \tau_{3}$ & $\Lambda_{z}, C T_{\perp}$ & $2 \times \mathrm{D}$ & $\theta=\pi$ & $4 e^{2} / \pi h$ \\
Charged impurities $+(\mathrm{RMF}$, ripples $)$ & $\Lambda_{z}$ & $2 \times \mathrm{A}$ & $\theta=\pi$ & $4 \sigma_{U}^{*}$ \\
\hline \hline
\end{tabular}

can have only off-diagonal matrix elements in the AB space of sublattices [18. Away from the Dirac point $(\varepsilon \neq 0)$, any chiral symmetry is broken by the diagonal $\left(\sigma_{0} \tau_{0}\right)$ energy term in the action. On the other hand, at half filling the chiral symmetry plays crucial role in establishing the transport properties of the system, see Sec. 3. If disorder commutes with $\Lambda_{z}$ (long-range), the system splits into two copies (decoupled valleys). The conductivity in this case is discussed in Sec. 4

In Table 2 we list all possible situations when the symmetry prevents the localization and leads to a finite conductivity at $\varepsilon=0$. This happens if the system possesses chiral symmetry or the valleys are decoupled, as we describe in detail below. 


\section{Chiral disorder}

The special class of disorder that we will consider in this section is the randomness that preserves one of the chiral symmetries of the clean graphene Hamiltonian [16]. Some possible realizations of such type of disorder are listed in Table 2. A peculiar behavior of 2D systems with chiral disorder with respect to localization effects has been demonstrated by Gade and Wegner 2324 . They considered a random hopping problem on a square lattice and showed that at zero energy, where the system possesses the chiral symmetry, the RG $\beta$-function of the corresponding sigma model vanishes to all orders in the inverse conductivity, implying that the conductivity is not renormalized. This absence of usual infrared-singular corrections to the conductivity due to Cooperon and diffuson loops can be attributed to the fact that the "antilocalizing" interference corrections to the density of states cancel the localization corrections to the diffusion coefficient. The states at the band center $\varepsilon=0$ are delocalized and the conductivity $\sigma(\varepsilon=0)$ takes a finite value depending on the disorder strength. According to the classification of Refs. [192021, the system studied in Refs. [2324] belongs to the chiral orthogonal symmetry class BDI if the time-reversal symmetry is preserved and to the chiral unitary symmetry class AIII otherwise. The peculiarity of the problem we are considering is the Dirac dispersion of carriers. This will allow us to prove below a statement that is still stronger than that of Gade and Wegner: we will show that for certain types of chiral disorder all disorder-induced contributions to conductivity cancel and $\sigma(\varepsilon=0)$ takes the universal value $4 e^{2} / \pi h$.

\section{1 $C_{0}$ chirality}

Let us consider the disorder which preserves the $C_{0}$-chirality, $H=-\sigma_{3} H \sigma_{3}$. The random part of the Hamiltonian contains matrices $\sigma_{1,2} \tau_{3}, \sigma_{1,2} \tau_{1,2}$, and $\sigma_{1,2} \tau_{0}$. According to Ref. [27, the random Dirac Hamiltonians preserving the $C_{0}$ chirality and violating the $T_{0}$ symmetry belong to the chiral symmetry class AIII, while the combination of $C_{0}$ chirality and $T_{0}$ symmetry drives the system into the Bogolyubov - de Gennes symmetry class CI. In both cases, the low-energy theory is affected [2821] by the presence of the Wess-Zumino-Witten term [25] in the action.

Let us turn to the calculation of the conductivity at half filling, assuming the $C_{0}$ disorder. The conductivity is given by the Kubo formula

$$
\sigma=-\frac{1}{2 \pi \hbar} \operatorname{Tr}\left[j^{x}\left(G^{R}-G^{A}\right) j^{x}\left(G^{R}-G^{A}\right)\right] .
$$

Here 'Tr' operation implies matrix trace and the spatial integration. Now we use the identity

$$
\sigma_{3} G^{R(A)}(\varepsilon) \sigma_{3}=-G^{A(R)}(-\varepsilon)
$$

valid when disorder preserves $C_{0}$-chirality. This allows us to trade all advanced Green functions in Eq. (44) for retarded ones and thus to present the conductivity at zero energy in terms of retarded Green functions. Further, we exploit the following important relation between the components of the current operator

$$
\sigma_{3} j^{x}=-j^{x} \sigma_{3}=i j^{y}
$$

which is the consequence of the Dirac spectrum. At this point, our problem differs from that considered by Gade and Wegner [23 24] who dealt with a bipartite square lattice with a nonlinear electronic spectrum.

The transformations Eqs. (5) and (6) allow us to cast the Kubo formula in the following form:

$$
\sigma(\varepsilon=0)=-\frac{1}{\pi \hbar} \sum_{\alpha=x, y} \operatorname{Tr}\left[j^{\alpha} G^{R} j^{\alpha} G^{R}\right] .
$$

At first glance, this expression is zero due to gauge invariance. Indeed, the right-hand side of Eq. (17) is proportional to the second derivative of the partition function $Z[\mathbf{A}]=\operatorname{Tr} \log G^{R}[\mathbf{A}]$ 
(or, equivalently, first derivative of the current $\operatorname{Tr} j^{\alpha} G^{R}[\mathbf{A}]$ ) with respect to the constant vector potential A. The gauge invariance implies that a constant vector potential does not affect gauge-invariant quantities like the partition function or the current, so that the derivative is zero. This argument is, however, not correct for the zero-order diagram (the one with no disorder included), in view of the quantum anomaly [2930]. The explicit calculation then yields

$$
\sigma=-\frac{8 e^{2} v_{0}^{2}}{\pi \hbar} \int \frac{d^{2} k}{(2 \pi)^{2}} \frac{\delta^{2}}{\left(v_{0}^{2} k^{2}+\delta^{2}\right)^{2}}=\frac{4 e^{2}}{\pi h} .
$$

Here $\delta$ is an infinitesimal imaginary part in the denominator of the Green function. Physically, it has a meaning of the electron lifetime or, alternatively, a dephasing rate, and can be thought of as modelling processes of escape of electrons in some reservoir or some dephasing mechanism. Models with such a uniform constant value of $\delta$ were used in the literature to imitate dephasing in quantum dots, see e.g. Ref. [31. The corrections to Eq. (8) are exponentially small in the disorder strength.

We note that the same universal value of the conductivity in the situation when the only type of disorder is the Abelian random vector potential was previously obtained in Ref. 32; for a certain type of the non-Abelian gauge potential with Gaussian distribution, universal conductivity was also obtained in Ref. 33. The above derivation of the universal conductivity remains valid for the case when a magnetic field of an arbitrary strength is applied: the vector potential A couples to the current, i.e. to the matrices $\tau_{3} \sigma_{1,2}$, thus preserving the chiral symmetry. In this context, it is worth mentioning the result of Hikami, Shirai, and Wegner 34 who found that the longitudinal conductivity in the center of the lowest Landau level of the chiral-disordered $2 \mathrm{D}$ electron gas is equal exactly to $\sigma=4 e^{2} / \pi h$ in the limit of very strong magnetic field, when the Landau level mixing can be neglected.

\section{2 $C_{z}$ chirality}

In this subsection we consider the disorder which preserves the $C_{z}$ chirality, $H=-\sigma_{3} \tau_{3} H \sigma_{3} \tau_{3}$. The random part of the Hamiltonian may then contain matrices $\sigma_{3} \tau_{1,2}, \sigma_{1,2} \tau_{3}, \sigma_{1,2} \tau_{0}$, and $\sigma_{0} \tau_{1,2}$. Random Dirac Hamiltonians [27. preserving the $C_{z}$ chirality and violating the $T_{0}$ symmetry belong to the chiral unitary symmetry class AIII. WZW terms 25] from the two valleys cancel out [21] in this case. The combination of $C_{z}$ chirality and the time reversal invariance $T_{0}$ corresponds to the chiral orthogonal symmetry class BDI. Finally, the combination of $C_{z}$ chirality and $T_{z}$ symmetry falls into the chiral symplectic symmetry class CII. In all these situations, the resulting theory is of the Gade type 2324].

Let us turn to the conductivity at half filling for a generic disorder preserving the $C_{z}$ chirality. The proof of the universality of the conductivity based on gauge-invariance argument does not work now since the $C_{z}$ chirality transformation of the Green's function generates the new vector vertices $j^{x, y} \tau_{3}$ instead of physical currents. Nevertheless, for weak disorder we find that the conductivity at half filling is still universal, $\sigma(\varepsilon=0)=4 e^{2} / \pi h$, up to corrections in powers of disorder strength (see also [35]). To show this, we first calculate the perturbative correction $\delta \sigma^{(1)}$ to the conductivity of a pure system at the first order in disorder strength and find that it vanishes, $\delta \sigma^{(1)}=0$. This implies that the conductivity at the Dirac point does not depend on the ultraviolet cut-off of the theory and can be presented as a series in the weak disorder strength (indeed the second-order term is found to be finite) 16. Next, we recall that for $C_{z}$ chirality, the RG $\beta$-function of the Gade-Wegner sigma model [2324] vanishes to all orders, so that there are no singular quantum-interference corrections to $\sigma(\varepsilon=0)$ due to the soft modes (impurity ladders). This proves that the expansion of $\sigma(\varepsilon=0)$ in powers of disorder converges. Thus for the case of weak disorder the conductivity is universal with small corrections in powers of the disorder strength (unlike in the case of the $C_{0}$ chirality, where the corrections are nonperturbative in the disorder strength.)

Finally, let us mention the case of $C_{\perp}$ chirality. In an isotropic system considered here, both $C_{x}$ and $C_{y}$ chiralities are expected to be present simultaneously. This implies that the disordered Hamiltonian anticommutes with both $\tau_{1}$ and $\tau_{2}$ and hence is proportional to $\tau_{3}$. 
Thus it is split into two equivalent copies. This situation of decoupled valleys corresponds to the case of long-range disorder and will be considered in Sec. 4 .

\subsection{Conductivity at finite frequency or finite temperature}

In this subsection, we analyze the frequency dependence of the conductivity in the case of chiral disorder. For completeness, we also keep a small level width $\delta$ introduced above. It was crucial for the argument leading to Eq. (8) that the system is exactly at half filling, $\varepsilon=0$. A non-zero frequency implies an integration over the energy range of the width $\omega$, which breaks the chiral symmetry. When the frequency $\omega$ is much smaller than $\delta$, this effect is however negligible, the infrared regularization is provided by $\delta$, and the universal result (8) survives. In its turn, $\delta$ plays no role when $\omega \gg \delta$ : it is the frequency that serves as a dominant infrared cutoff now. Then a very interesting new situation arises since $\omega$ plays a twofold role, leading to two competing effects. On one hand, as discussed above, the frequency drives the system away from the chiralsymmetric point and thus restores localization. On the other hand, the frequency cuts off the singular localization correction. Which of these effects wins? To answer this question, one should compare $\omega$ with the level spacing in the localization area, $\Delta_{\xi}(\varepsilon)$, where $\varepsilon \sim \omega$. It turns out that $\Delta_{\xi}(\varepsilon) \sim \varepsilon \sim \omega$ for the chiral disorder. This result is rather general and is only based on the fact that the operator governing the flow of the system away from criticality couples to the energy in the action. Therefore the two competing effects of the frequency (the localization and the infrared regularization) "make a draw" - both of them are equally important. The system turns out to be, roughly speaking, half way between the chiral fixed point and the conventional symmetry. This results in a new universal (frequency-independent) value of the conductivity $\sigma_{\omega} \sim e^{2} / h$ in the considered regime $\delta \ll \omega$. More precisely, this value depends on the type of chirality and the symmetry class of the system away from the Dirac point.

In the presence of interactions, the temperature $T$ also plays a twofold role, similarly to the frequency. On one hand, it induces an averaging over the energy window of the width $\sim T$, thus breaking the chiral symmetry and "switching on" the localization effects. On the other hand, the interaction at finite $T$ generates a non-zero dephasing rate $\tau_{\phi}^{-1}(T)$ cutting off the localization corrections. As we showed above, the level spacing $\Delta_{\xi}(T)$ is $\sim T$, so that the result of the competition of these two effects depend on the value of $T \tau_{\phi}(T)$. It is natural to assume that $\tau_{\phi}^{-1}(T) \sim T$ [13]16], as follows from the result of Ref. [36] for a two-dimensional diffusive metal with Coulomb interaction at $\sigma \sim e^{2} / h$. Then the behavior of the conductivity at low $T$ will be qualitatively analogous to that for the case of finite frequency.

More realistically, one can think about a situation when the disorder is predominantly chiral (say, due to ripples and dislocations), but the chiral symmetry is slightly broken, e.g., by weak short-range potential disorder. Then the above consideration will be applicable in the parametrically broad range of $T$; at the lowest temperatures, the chirality-breaking effects will drive the system into the strong localization regime.

\section{Long-range disorder}

In the previous section we have shown that, if one of the chiral symmetries of clean graphene is preserved by disorder, the conductivity at half filling is not affected by localization and is equal to $4 e^{2} / \pi h$. While various types of randomness in graphene (in particular, dislocations, ripples, or strong point-like defects) do belong to the chiral type, the experimentally observed value of $\sigma$ is larger by a factor $\sim 3$, suggesting a different type of criticality. In this section we consider another broad class of randomness in graphene - long-range disorder 17 — commuting with $\Lambda_{z}$ (see Table 1). We will show the possibility of a new critical state when valleys are decoupled. This case has a particular experimental relevance if the conductivity is dominated by charged impurities. The ripples 3738 belong to this type of randomness as well. Numerical simulations of graphene with long-range random potential [39]40] provide an evidence in favor of a scaleinvariant conductivity. 
The characteristic feature of the long-range disorder is the absence of valley mixing due to the lack of scattering with large momentum transfer. This allows us to describe the system in terms of a single-valley Dirac Hamiltonian with disorder [32 28,41,

$$
H=v_{0} \boldsymbol{\sigma} \mathbf{k}+\sigma_{\mu} V_{\mu}(\mathbf{r}) .
$$

Here disorder includes random scalar $\left(V_{0}\right)$ and vector $\left(V_{1,2}\right)$ potentials and random mass $\left(V_{3}\right)$. The Hamiltonian (9) was considered in Ref. 32 as a model for quantum Hall transition.

The clean single-valley Hamiltonian (9) obeys [3242] the effective time-reversal invariance $H=\sigma_{2} H^{T} \sigma_{2}$. This symmetry $\left(T_{\perp}\right)$ is not the physical time-reversal symmetry $\left(T_{0}\right)$ : the latter interchanges the nodes and is of no significance in the absence of inter-node scattering. Below we refer to the effective time-reversal symmetry in a single node as the TR symmetry. If the only disorder is random scalar potential, the TR invariance is not broken and the system falls into the symplectic symmetry class AII, see Refs. 3213 14,12. The standard realization of such symmetry is a system with spin-orbit coupling; in the present context the role of spin is played by the sublattice space.

\subsection{Unitary class}

We start with a more generic case of the unitary symmetry (class A). The TR invariance is broken as soon as a (either random or non-random) mass or vector potential is included, in addition to the scalar potential. To analyze the critical behavior of the system, we derive the nonlinear sigma model for the single-node Dirac fermions [17] at finite energy $\varepsilon$. This model is formulated in terms of the $4 \times 4$ supermatrix field $Q$ operating in Bose-Fermi (BF) superspace and bearing the retarded-advanced (RA) structure [31]. The sigma-model action reads [17]

$$
S[Q]=\frac{1}{4} \operatorname{Str}\left[-\frac{\sigma_{x x}}{2}(\nabla Q)^{2}+\left(\sigma_{x y}+\frac{1}{2}\right) Q \nabla_{x} Q \nabla_{y} Q\right] \equiv S_{1}[Q]+i S_{2}[Q]
$$

Here 'Str' operation includes the matrix supertrace and the spatial integration. The parameters $\sigma_{x x}$ and $\sigma_{x y}$ of the action are the longitudinal and Hall conductivities (in units $e^{2} / h$ ) given by the corresponding Kubo expressions for a single node. In the presence of the constant mass $m$, they acquire the values

$$
\begin{gathered}
\sigma_{x x}=\frac{1}{2 \pi}\left[1+\frac{\varepsilon^{2}+\gamma^{2}-m^{2}}{2 \gamma} f(\varepsilon, m)\right], \quad \sigma_{x y}=-\frac{m}{2 \pi}[f(\varepsilon, m)+f(m, \varepsilon)] \\
f(x, y)=\frac{1}{x}\left[\arctan \frac{x+y}{\gamma}+\arctan \frac{x-y}{\gamma}\right]
\end{gathered}
$$

where $\gamma$ is the elastic scattering rate. Note that if the global $T_{0}$ symmetry involving both the valleys is preserved, the observable Hall conductivity is absent due to the cancellation between the contributions of the two valleys.

The imaginary part of the action, $i S_{2}[Q]$, is determined by the well-known topological invariant $\operatorname{Str}\left(Q \nabla_{x} Q \nabla_{y} Q\right) \equiv 8 i \pi N[Q]$ on the sigma-model manifold [26. Possible values of this functional are integer multiples of $8 \pi i$. In Eq. (10), the topological term is equal to $i \theta N[Q]$, with the angle $\theta=2 \pi \sigma_{x y}+\pi$. In graphene the mass $m$ is absent, so that $\sigma_{x y}=0$. Thus, the topological angle is $\theta=\pi$. The theory (10) is then exactly on the critical line of the quantum Hall transition 2643, in agreement with the arguments of Ref. 32. We thus conclude that graphene with a generic (TR-breaking) long-range disorder is driven into the quantum Hall critical point (i.e. we have a "quantum Hall effect without Landau levels" 44), with the conductivity $4 \sigma_{U}^{*}$. The factor 4 here accounts for the spin and valley degeneracy. The value $\sigma_{U}^{*}$ is known to be in the range $\sigma_{U}^{*} \simeq 0.5-0.6 e^{2} / h$ from numerical simulations [45. A schematic scaling function in this case is shown in Fig. 17. While formally this conclusion holds for any energy $\varepsilon$, in reality it only works near half filling; for other $\varepsilon$ the quantum Hall critical point would only be reached for unrealistic temperatures and system sizes. It is worth noting that the sigma 

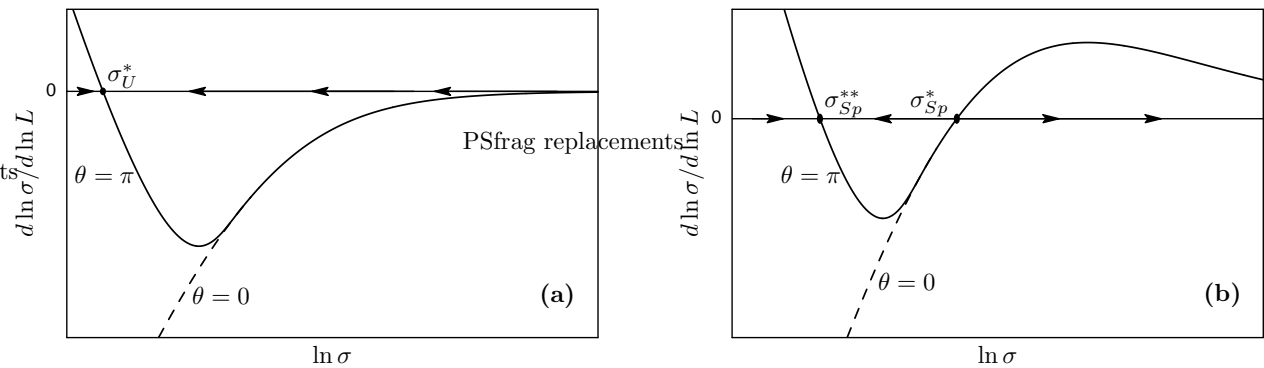

Fig. 1. Schematic scaling functions for (a) unitary and (b) symplectic universality class with topological term $\theta=\pi$. The ordinary case $\theta=0$ is shown by dashed lines.

model Eq. (10) is rigorously derived only for $\sigma_{x x} \gg 1$, whereas $\sigma_{x x} \sim 1$ at $\varepsilon=0$. This should not, however, affect the universal critical behavior of the theory governed by symmetry and topology of the problem. An alternative route from the Dirac anomaly to a topological term employs non-Abelian bosonization, see Refs. 2141.

If a uniform transverse magnetic field is applied, the topological angle $\theta$ becomes energydependent. However, at zero energy, where $\sigma_{x y}=0$, its value remains unchanged, $\theta=\pi$. This implies the emergence of the half-integer quantum Hall effect, with a plateau transition point at $\varepsilon=0$. An alternative explanation [5] of the half-integer quantum Hall effect is based on the concept of the Berry phase $\pi$ in graphene [42]. This relates the Berry phase and the anomalous topological angle $\theta=\pi$.

In the above, we have assumed a generic TR-breaking long-range disorder. For specific kinds of disorder, additional $C$ or $C T$ symmetries may emerge at zero energy. In particular, if the only disorder is a random vector potential (ripples), the system belongs to the chiral class AIII, with $\sigma=4 e^{2} / \pi h$, see Sec. 3. If only the random mass is present, the system falls into class D at $\varepsilon=0$. In this case the weak disorder is known to be irrelevant [32 2841], implying that the conductivity is again $4 e^{2} / \pi h$.

\subsection{Symplectic class}

Let us now turn to the case of preserved TR invariance, describing in particular charged impurities. The system belongs then to the symplectic symmetry class AII. The derivation of the $\sigma$-model starts with the doubling of $\psi$ variables accounting for the TR symmetry [31]. Then $Q$ is a $8 \times 8$ matrix obeying an additional constraint of charge conjugation $Q=\bar{Q}$.

Since the partition function of the symplectic model is real, the imaginary part of the action $S_{2}$ can take one of the two possible values, 0 or $\pi$. The discreteness of $S_{2}$ suggests that it again should be proportional to a topological invariant on the sigma-model manifold. A non-trivial topology may arise only in the compact (fermion) sector of $Q$. The corresponding target space is $\mathcal{M}_{F}=O(4 n) / O(2 n) \times O(2 n)$, where $n$ is the number of fermion species. While for the conventional (sufficient for the analysis of conductivity) sigma model $n=1$, larger values will arise if one considers higher-order products of Green functions. The topological invariant takes values from the homotopy group 46.

$$
\pi_{2}\left[\left.\mathcal{M}_{F}\right|_{n=1}\right]=\mathbb{Z} \times \mathbb{Z}, \quad \pi_{2}\left[\left.\mathcal{M}_{F}\right|_{n \geq 2}\right]=\mathbb{Z}_{2}
$$

The homotopy group in the case $n=1$ is richer than for $n \geq 2$. Nevertheless, $S_{2}$ may take only two non-equivalent values. Hence only a certain $\mathbb{Z}_{2}$ subgroup of the whole $\mathbb{Z} \times \mathbb{Z}$ comes into play as expected: the phase diagram of the theory should not depend on $n$. Possibility of the $\mathbb{Z}_{2}$ topological term in the $2 \mathrm{D}$ symplectic sigma model was emphasized by Fendley in Refs. 2247 .

To demonstrate the emerging topology explicitly and to calculate the topological invariant, let us analyze the case $n=1$ in more detail. The generators of the compact sector are Hermitian skew-symmetric $4 \times 4$ matrices anticommuting with $\Lambda \equiv \rho_{3}: \rho_{1} \mu_{2}, \rho_{2} \mu_{0}, \rho_{2} \mu_{1}$, and $\rho_{2} \mu_{3}$. Here $\rho_{i}$ and $\mu_{i}$ are Pauli matrices in RA and TR space respectively. These generators split into two 
mutually commuting pairs, each generating a 2-sphere ("diffuson" and "Cooperon" sphere). Simultaneous inversion of both spheres leaves $Q$ intact. Hence the compact sector of the model is the manifold $\left(\mathcal{S}_{2} \times \mathcal{S}_{2}\right) / \mathbb{Z}_{2}$. Thus two topological invariants, $N_{1,2}[Q]$, counting the covering of each sphere, emerge in accordance with Eq. (13). The most general topological term is $\theta_{1} N_{1}+\theta_{2} N_{2}$. Due to the TR symmetry, the action is invariant under interchanging the diffuson and Cooperon spheres, which yields $\theta_{1}=\theta_{2} \equiv \theta$ where $\theta$ is either 0 or $\pi$. The explicit calculation of the $n=1$ topological term yields

$$
i S_{2}[Q]=\frac{\epsilon_{\alpha \beta}}{8} \operatorname{Str}\left[(\Lambda \pm 1) \mu_{2} u_{\alpha} u_{\beta}\right]
$$

where $\mathbf{u}=T \nabla T^{-1}$ and $Q=T^{-1} \Lambda T$. The sign ambiguity here does not affect any observables. Equation (14) can be cast in the form $i S_{2}[Q]=i \pi\left(N_{1}[Q]+N_{2}[Q]\right)$, thus yielding $\theta=\pi$. If the TR symmetry is broken, the Cooperon modes are frozen and the manifold is reduced to a single diffuson sphere $\left(Q\right.$, and hence $\mathbf{u}$, commutes with $\left.\mu_{2}\right)$ with $i S_{2}=i \pi N_{1}[Q]$. In this case, $N_{1}[Q]=\left(\epsilon_{\alpha \beta} / 8 i \pi\right) \operatorname{Str}\left(\Lambda \mu_{2} u_{\alpha} u_{\beta}\right)=(i / 16 \pi) \operatorname{Str}\left(Q \nabla_{x} Q \nabla_{y} Q \mu_{2}\right)$. In the case $n>1$, the topological term is $i S_{2}[Q]=i \pi N[Q]$ with $N[Q] \in \mathbb{Z}_{2} \equiv\{0,1\}$, cf. Eq. (13). The statement $\theta=\pi$ follows from the fact that $Q$ configurations of the $n=1$ theory which have an odd topological charge $N[Q]$ and yield a negative partition function, are inherited by the $n>1$ theory by virtue of natural embedding of the corresponding manifolds. The explicit form Eq. (14) is, however, not applicable in the $n>1$ case, since the manifold no longer decomposes into two spheres.

An ordinary symplectic theory with no topological term exhibits metal-insulator transition at $\sigma_{S p}^{*} \approx 1.4 e^{2} / h$ [8]. If the conductivity is smaller than this critical value, the localization drives the system into insulating state, while in the metallic phase, $\sigma>\sigma_{S p}^{*}$, antilocalization occurs. Using the analogy with the quantum Hall transition in the unitary class [26 43], we argue that the topological term with $\theta=\pi$ suppresses localization effects when the conductivity is small, leading to appearance of a new attracting fixed point at $\sigma_{S p}^{* *}$. The position of the metalinsulator transition, $\sigma_{S p}^{*}$, is also affected by the topological term. However, we believe that its change is negligible: the instanton correction to the scaling function at large conductivity is exponentially small [26], and the value of the exponential factor $e^{-2 \pi \sigma}$ is still extremely small at $\sigma=\sigma_{S p}^{*}$. A plausible scaling of the conductivity in the symplectic case with $\theta=\pi$ is sketched in Fig. 1 b. The existence and position of the new critical point can be verified numerically. Recent simulations of graphene 3940] indeed demonstrate the stability of the conductivity in the presence of long-range disorder. Of course, in reality there will be always a weak inter-valley scattering, which will establish the localization at lowest $T$, in agreement with 1314. However, the approximate quantum criticality will hold in a parametrically broad range of $T$.

Before concluding, let us briefly discuss this crossover to localization in graphene with charged impurities. The dominant diagonal $\left(\sigma_{0} \tau_{0}\right)$ scattering amplitude does not participate in the ultra-violet renormalization [32 281316], in contrast to the case of a finite-range intra-valley disorder. The reason is the long-range type of correlations $\left(\propto q^{-2}\right.$ in momentum space) that resulted in the absence of ultra-violet corrections to the linear conductivity in Refs. [49391650]. Near half filling, the scattering rate is $\gamma \sim h v_{0} n_{\text {imp }}^{1 / 2}$, where $n_{\text {imp }}$ is the concentration of Coulomb impurities and the interaction parameter $r_{s}$ is set to unity. The inter-valley processes occur with a much smaller rate $\gamma^{\prime} \sim a^{2} n_{\text {imp }} \gamma$, where $a$ is the lattice constant. Note that the value of $\gamma^{\prime}$ is only weakly corrected due to ultra-violet renormalization, unlike in the model of white-noise disorder [13. Assuming as above $\tau_{\phi}^{-1} \sim T$, the crossover to strong localization (orthogonal symmetry class AI) takes place at temperatures of the order of $\gamma^{\prime}$. Using realistic parameters from Ref. [5], we find that the symplectic quantum criticality characterized by a scale-invariant conductivity $\sigma_{S p}^{* *}$ holds down to $10-50 \mathrm{mK}$. Therefore, the model of long-range charged impurities is capable of describing simultaneously the two most striking experimental observations [56]: the linear density dependence of the conductivity [4939] and the existence of a universal minimal conductivity at the Dirac point [17 in a broad temperature range. 


\section{Conclusions}

To summarize, we have studied electron transport properties of a disordered graphene layer. We have shown that the nature of disorder is of crucial importance for the behavior of the conductivity. At half filling, the conductivity is of the order of $e^{2} / h$ if the randomness preserves one of the chiral symmetries of the clean Hamiltonian or does not mix the valleys.

For the case of chiral disorder, the exact value of the conductivity is still determined by the nature of the infrared cutoff, which may depend on the physical setup. We have analyzed in detail the situation when this cutoff is provided by the level width $\delta$ or by the frequency $\omega$. In the former case the conductivity takes a universal value $4 e^{2} / \pi h$, while in the latter case it shows a more complex behavior still being of the order of conductance quantum.

In the case of the long-range (no inter-valley scattering) disorder, graphene also shows quantum criticality at half filling. If the effective TR symmetry of the single-valley system is preserved (e.g. when Coulomb scatterers are the dominant disorder), the relevant theory is the symplectic sigma model with topological angle $\theta=\pi$ and the minimal conductivity takes the universal value $4 \sigma_{S p}^{* *}$. If the TR symmetry is broken (e.g. by effective random magnetic field

due to ripples or by external magnetic field), the system falls into the universality class of the quantum Hall critical point, with another universal value $4 \sigma_{U}^{*}$.

\section{Acknowledgments}

We thank V. Cheianov, D.I. Diakonov, F. Evers, A. Geim, I.A. Gruzberg, H. von Löhneysen, A.W.W. Ludwig, Y. Makhlin, S.V. Morozov, A.F. Morpurgo, C. Mudry, K.S. Novoselov, S. Ryu, V. Serganova, M.A. Skvortsov, A.G. Yashenkin, and I. Zakharevich for valuable discussions and comments. The work was supported by the Center for Functional Nanostructures and the Schwerpunktprogramm "Quanten-Hall-Systeme" of the Deutsche Forschungsgemeinschaft. The work of PMO was supported by the Russian Foundation for Basic Research under grant No. 04-0216348 and by the Russian Academy of Sciences under the program "Quantum Macrophysics." ADM acknowledges hospitality of the Kavli Institute for Theoretical Physics at Santa Barbara and partial support by the National Science Foundation under Grant No. PHY99-07949. The work of IVG, conducted as a part of the project "Quantum Transport in Nanostructures" made under the EUROHORCS/ESF EURYI Awards scheme, was supported by funds from the Participating Organizations of EURYI and the EC Sixth Framework Programme. IVG is grateful to the organizers of the workshop COQUSY06 for a friendly and stimulating atmosphere during the Graphene Conference and acknowledges the hospitality of the Max Planck Institute for the Physics of Complex Systems in Dresden. IVG is also indebted to the museum guide of the COQUSY06-excursion at the Green Vault in Dresden for his layman's remark: "Graphene? I know: this is like the quantum Hall effect!"

\section{References}

1. P.W. Anderson, Phys. Rev. 109, 1492 (1958).

2. E. Abrahams, P.W. Anderson, D.C. Licciardello, and T.V. Ramakrishnan, Phys. Rev. Lett. 42, 673 (1979).

3. L.P. Gorkov, A.I. Larkin and D.E. Khmelnitskii, Pis'ma Zh. Eksp. Teor. Fiz. 30, 248 (1979) [JETP Lett. 30, 228 (1979)].

4. K.S. Novoselov, A.K. Geim, S.V. Morozov, D. Jiang, Y. Zhang, S.V. Dubonos, I.V. Grigorieva, and A.A. Firsov, Science 306, 666 (2004).

5. K.S. Novoselov, A.K. Geim, S.V. Morozov, D. Jiang, M.I. Katsnelson, I.V. Grigorieva, S.V. Dubonos, and A.A. Firsov, Nature 438, 197 (2005).

6. Y. Zhang, Y.-W. Tan, H.L. Stormer, and P. Kim, Nature 438, 201 (2005).

7. K.S. Novoselov, E. McCann, S.V. Morozov, V.I. Falko, M.I.Katsnelson, U. Zeitler, D. Jiang, F. Schedin, and A.K. Geim, Nature Physics, 2, 177 (2006). 
8. S.V. Morozov, K.S. Novoselov, M.I. Katsnelson, F. Schedin, L.A. Ponomarenko, D. Jiang, and A.K. Geim, Phys. Rev. Lett. 97, 016801 (2006).

9. Y. Zhang, Z. Jiang, J.P. Small, M.S. Purewal, Y.-W. Tan, M. Fazlollahi, J.D. Chudow, J.A. Jaszczak, H.L. Stormer, and P. Kim, Phys. Rev. Lett. 96, 136806 (2006).

10. P.R. Wallace, Phys. Rev. 71, 622 (1947).

11. T. Ando, J. Phys. Soc. Jpn 74, 777 (2005).

12. E. McCann, K. Kechedzhi, V.I. Fal'ko, H. Suzuura, T. Ando, and B.L. Altshuler, Phys. Rev. Lett. 97, 146805 (2006).

13. I.L. Aleiner and K.B. Efetov, Phys. Rev. Lett. 97, 236801 (2006).

14. A. Altland, Phys. Rev. Lett. 97, 236802 (2006).

15. N.H. Shon and T. Ando, J. Phys. Soc. Jpn 67, 2421 (1998).

16. P.M. Ostrovsky, I.V. Gornyi, and A.D. Mirlin, Phys. Rev. B 74, 235443 (2006).

17. P.M. Ostrovsky, I.V. Gornyi, and A.D. Mirlin, cond-mat/0702115.

18. S. Guruswamy, A. LeClair, and A.W.W. Ludwig, Nucl. Phys. B 583, 475 (2000).

19. M.R. Zirnbauer, J. Math. Phys. 37, 4986 (1996).

20. A. Altland and M.R. Zirnbauer, Phys. Rev. B 55, 1142 (1997).

21. A. Altland, B.D. Simons, and M.R. Zirnbauer, Phys. Rep. 359, 283 (2002).

22. P. Fendley, in Proceedings of the NATO ASI on New Theoretical Approaches to Strongly Correlated Systems, Cambridge, UK, 2000, edited by A. Tsvelik (Kluwer Academic Publishers, Dordrecht, 2001), p. 141.

23. R. Gade and F. Wegner, Nucl. Phys. B 360, 213 (1991).

24. R. Gade, Nucl. Phys. B 398, 499 (1993).

25. E. Witten, Comm. Math. Phys. 92, 455 (1984).

26. A.M.M. Pruisken, Nucl. Phys. B 235, 277 (1984); in The Quantum Hall Effect ed. by R.E. Prange and S.M. Girvin (Springer, 1987), p. 117.

27. D. Bernard and A. LeClair, J. Phys. A 35, 2555 (2002).

28. A. A. Nersesyan, A. M. Tsvelik, and F. Wenger, Phys. Rev. Lett. 72, 2628 (1994); Nucl. Phys. B 438, 561 (1995).

29. J. Schwinger, Phys. Rev. 128, 2425 (1962).

30. M.E. Peskin and D.V. Schroeder, An Introduction to Quantum Field Theory (Addison-Wesley Advanced Book Program, Westview Press, Boulder, 1995).

31. K.B. Efetov, Supersymmetry in disorder and chaos (Cambridge University Press, Cambridge, 1996).

32. A.W.W. Ludwig, M.P.A. Fisher, R. Shankar, and G. Grinstein, Phys. Rev. B 50, 7526 (1994).

33. A.M. Tsvelik, Phys. Rev. B 51, 9449 (1995).

34. S. Hikami, M. Shirai, and F. Wegner, Nucl. Phys. B 408, 415 (1993).

35. S. Ryu, C. Mudry, A. Furusaki, and A.W.W. Ludwig, cond-mat/0610598.

36. B.L. Altshuler, A.G. Aronov, and D.E. Khmelnitsky, J. Phys. C 15, 7367 (1982).

37. A.F. Morpurgo and F. Guinea, Phys. Rev. Lett. 97, 196804 (2006).

38. J.C. Meyer, A.K. Geim, M.I. Katsnelson, K.S. Novoselov, T.J. Booth, and S. Roth, cond-mat/0701379.

39. K. Nomura and A.H. MacDonald, Phys. Rev. Lett. 98, 076602 (2007).

40. A. Rycerz, J. Tworzydlo, and C.W.J. Beenakker, cond-mat/0612446.

41. M. Bocquet, D. Serban, and M.R. Zirnbauer, Nucl. Phys. B 578, 628 (2000).

42. T. Ando, T. Nakanishi, and R. Saito, J. Phys. Soc. Jpn 67, 2857 (1998); H. Suzuura and T. Ando, Phys. Rev. Lett. 89, 266603 (2002); J. Phys. Soc. Jpn 72, 69 (2003).

43. D.E. Khmel'nitskii, Pis'ma Zh. Eksp. Teor. Fiz. 38, 454 (1983) [JETP Letters 38, 552 (1983)].

44. F.D.M. Haldane, Phys. Rev. Lett. 61, 2015 (1988).

45. Y. Huo, R.E. Hetzel, and R.N. Bhatt, Phys. Rev. Lett. 70, 481 (1993); B.M. Gammel and W. Brenig, ibid 73, 3286 (1994); Z. Wang, B. Jovanović, and D.-H. Lee, ibid 77, 4426 (1996); S. Cho and M.P.A. Fisher, Phys. Rev. B 55, 1637 (1997); L. Schweitzer and P. Markoš, Phys. Rev. Lett. 95, 256805 (2005).

46. D.B. Fuchs and O.Ya. Viro, Topology II, in series Encyclopaedia of Mathematical Sciences ed. by V.A. Rokhlin and S.P. Novikov, Vol. 24 (Springer, 2004); A. Chalcraft, "Homotopy groups of flag manifolds", http://www.scoriton.demon.co.uk/flags.html

47. P. Fendley, Phys. Rev. B 63, 104429 (2001); in Proceedings of the NATO ARW on Statistical Field Theories, Como, Italy, 2001, edited by A. Cappelli and G. Mussardo (Kluwer Academic Publishers, Dordrecht, 2002), p. 223.

48. P. Markoš and L. Schweitzer, J. Phys. A 39, 3221 (2006).

49. T. Ando, J. Phys. Soc. Jpn 75, 074716 (2006).

50. D.V. Khveshchenko, cond-mat/0611485 